\title{
BRIEF
}

\section{Examining Social Identities of Patient Diversity Through Cases Presented in a Therapeutics Course Series}

\author{
Jesse Upton, PharmD, Lynn Kassel, PharmD, Anisa Hansen, PharmD, Jared Butler, PharmD \\ Drake University, College of Pharmacy and Health Sciences, Des Moines, Iowa
}

Corresponding Author: Lynn Kassel, Drake University, College of Pharmacy and Health Sciences, 2507 University Ave., Cline Atrium 007, Des Moines, IA 50311. Tel: 515-271-1923. Email: Lynn.kassel@ drake.edu

Submitted April 21, 2021; accepted November 29, 2021; ePublished December 2021

Objective. The inclusion of social identities, such as race, ethnicity, sexual orientation, gender identity, ability,
spirituality, nationality, and socioeconomic status, within cases in didactic pharmacy education is an issue of significant
debate. In considering what and how much to include of these identities into didactic cases, the first step could be to
measure the current inclusion. The purpose of this study was to quantify the presence of these social identities in cases
presented to student pharmacists in a three-semester course series. Methods. This was a review of 144 cases presented in a three-semester pharmacotherapeutics course series. The primary objective was to quantify the inclusion of each social identity. The secondary objective assessed if the identities were needed to answer specific questions related to each case. Cases were reviewed by two independent study researchers, with a third impartial reviewer settling disagreements.

Results. Explicitly stating these identities was infrequent among the cases. Race was explicitly stated in $14.6 \%$ of cases $(\mathrm{n}=21)$. Gender identity was explicitly named in 2 cases $(1.4 \%)$, whereas nearly all cases implied gender through pronouns. Gender was necessary to answer case questions in approximately $20 \%$ of the cases $(n=27)$. Socioeconomic status, ability, sexual orientation, and nationality were infrequently named among all cases, $6.3 \%, 4.9 \%, 1.4 \%$, and $1.4 \%$, respectively.

Conclusion. This study found that these social identities were rarely explicitly stated in cases. In determining the next steps for integrating social identities, pharmacy education must first take stock of its current use of these identities. Keywords: pharmacy education, social identities, diversity, equity, inclusion, curriculum, health care education

\section{INTRODUCTION}

Shared decision making in medical practice has become the mainstay of treatment in health care, allowing treatment individualization. Individualizing care offered to each patient requires social determinants of health that utilize social identities (described in Table 1) and other patient-specific factors. ${ }^{1}$ The most commonly used of these identities were previously considered "The Big 8", but this nomenclature has fallen out of favor due to implied hierarchy. ${ }^{2}$ A lack of disclosure or use of these identities has been linked with poorer health outcomes. ${ }^{3,4,5,6}$ Therefore, early exposure to social identities may help students understand their potential impact in practice.

Literature surrounding social identities has increased in recent years, and health care providers are paying more attention to the lens used to examine identities. ${ }^{7,8}$ The American Medical Association's (AMA) recently published guidance for reporting race and ethnicity within medical research may provide a framework in which educational cases can be viewed as well. ${ }^{9}$ The AMA intends to provide guidance on nomenclature and use of other social identities, such as sexual orientation, sex and gender, and socioeconomic status. ${ }^{9}$

As organizations consider the language used and how patient-specific information is presented in practice, an initial step in health care provider education may be to determine how or if this information is presented to learners in current curricula. In recent years, medical students have called for inclusion of social identities within their curricula to better prepare for practice. ${ }^{3,4,6}$ Others have voiced concern that identities are strongly embedded in one case or example and are not expected to be considered throughout the curriculum. ${ }^{10}$ The inclusion of social identities within other disciplines' curricula has been largely driven by students, staff, faculty, and community members, though administrative leaders are supportive of this. ${ }^{3}$ Some programs excel at integrating the content when a champion or perceived expert in the area leads the way. ${ }^{6}$ In order to consider the next steps with integration of diversity, equity, and inclusion into pharmacy curricula, programs must first measure their current inclusion of the social identities within the didactic setting. The purpose of this study was to quantify the presence of these eight social identities within patient cases presented to a single cohort of student pharmacists over a three-semester pharmacotherapy course series. 


\section{METHODS}

This was an observational study that examined patient cases from a three-semester pharmacotherapeutics course that utilized team-based learning (TBL) pedagogy. The cases were presented to one cohort of student pharmacists at a private, Predominantly White Institution (PWI) in the Midwest between January 2019 and May 2020. Faculty at the institution are predominantly White, and most of the clinical practice faculty identify as White. All patient cases were individually analyzed and reviewed for each of the previously listed social identities after presentation to students. No attempts were made to modify the inclusion of the identities while actively being taught. Cases were assessed by the investigators between January 2020 - May 2020. IRB approval was not required for this study, as patient cases were examined.

Each case underwent two independent reviews by study authors. One investigator provided consistency by reviewing all cases. The cases were equally divided amongst other investigators for a second review. A third, impartial reviewer settled any discrepancies of the categorization. Two of the investigators taught within the course series and recused themselves of assessing their own cases to avoid bias. A Qualtrics survey was used to record data for each case: disease state or topic area, the course in which the case was presented, and identification of primary and secondary outcomes. The survey provided a space for consistent reporting of the variables, decreasing variation between reviewers. The authors developed the data collection tool, as no known validated tool measured the desirable data points.

The primary objective of the study was to quantify the inclusion of each social identity. To meet the primary objective, the case must have explicitly stated, not implied, a specific social identity. The secondary objective focused on if each identity was needed to answer specific case questions. Analysis utilizing descriptive statistics was completed using Microsoft Excel.

\section{RESULTS}

A total of 144 patient cases were presented to a single cohort of student pharmacists over a three semester pharmacotherapeutics course series between January 2019 and May 2020. Volume of cases presented between courses were 41, 65, and 38, which covered 19, 20, and 22 topics, respectively. Per topic, the mean number of patient cases presented was $2.6(\mathrm{SD}=1.7)$, with a mode of two cases and a range of one to eight cases.

The primary outcome showed that only 39 cases $(27.1 \%)$ explicitly stated any of the examined social identities. Thirty-five cases $(24.3 \%)$ explicitly stated one identity and four cases $(2.8 \%)$ explicitly stated more than one identity within the case. Race was the most often included social identity. Named races included: White $(52.4 \%, \mathrm{n}=11)$, Black $(42.9 \%, \mathrm{n}=9)$, and Hispanic $(4.8 \%, \mathrm{n}=1)$. Further description of the primary and secondary outcomes is presented in Table 2 .

\section{DISCUSSION}

Overall, cases presented within a three semester pharmacotherapeutic course series lacked explicit inclusion of any of the eight social identities reviewed.

The reported race of patients in these cases differed widely from that of the institution's state and city census data $^{11,12}$; however, the inclusion of a more diverse population in the classroom or objective structured clinical exam (OSCE) cases may be preparing learners to work with a broader patient population. These cases may present the opportunity for learners to further investigate specific identities that may alter the care plan. Moving forward from current practices of identity inclusion may resonate and lead to further learning and incorporation into clinical practice, as was seen in an OSCE related to limited English proficiency patients. ${ }^{13}$ The discrepancy in racial mismatching is not uniquely demonstrated solely within our curriculum; a study of a medical student preparatory question bank demonstrated that almost $86 \%$ of racial mentions were White, whereas entire populations (ie, Native Hawaiian/Pacific Islander) were excluded, ignoring the diversity within the United States. ${ }^{14}$ While most racial mentions were of patients who are White, race/ethnicity were only mentioned in approximately $20 \%$ of case questions $(n=455 / 2011)$ in the bank. ${ }^{14}$ This imbalance could potentially further biases, especially as non-White races were mentioned more frequently when they were necessary to answer the case questions. ${ }^{14}$ Lack of consistent inclusion of identities, particularly race, in patient presentations may result in the identity being labeled a distractor and may further instill implicit biases. ${ }^{15}$ Inclusion of specific identities should be balanced with inundating students with information, as a way to avoid instilling biases. When relevant to the care or case, race or other social identities may be best placed in the physical exam or social history instead of the introductory sentence. ${ }^{15}$

Behind race, socioeconomic status was the most included social identity, with the likely intent to consider cost in therapeutic decisions. Inclusion of socioeconomic status may further discussion regarding patient circumstances, such as employment, housing status, and food security, that would encourage practitioners to change care plans. Socioeconomic 
status and race are closely intertwined, as mentioning race could serve as a springboard to other discussions regarding influential elements of social determinants of health, such as education, food security, housing, employment opportunities, and health care access. ${ }^{7}$ Health professional students are expected to master content knowledge, communication skills, and connection with patients, ${ }^{5}$ thus raising the stakes when social identities are considered and need addressed. Purposeful consideration of the application of each identity is critical to remove barriers for equitable health care. Identifying and utilizing the intersections of identities among students, instructors, and patients ${ }^{16}$ is necessary to break down biases and further ingrain the identities within health care education. As shown in this study, the examined cases provided few opportunities to utilize and view these intersections.

Gender identity and sexual orientation were often implied, using pronouns or referring to a wife or boyfriend. The lack of inclusion could indicate discomfort with integrating the explicit identity into patient cases, or more likely: that it does not impact clinical decision making for most disease states. There is potential for heterosexual and binary gender identities to be the reinforced default if variations of those identities are not explicitly included. Their inclusion can be integrated into specific cases, stand-alone courses, or clinical rotations, ${ }^{6}$ demonstrating that there are multiple methods of exposure to prepare learners in their pathways to direct patient care.

When appropriate and intentional in nature, authentic inclusion of various social identities is critical to build informed and unbiased presentations to learners. Including those with lived experiences enhances authenticity, though involvement must be balanced with "tokenizing" that individual and their experiences for a specific case. ${ }^{3}$ A case may accurately represent a particular patient or encounter, though learners could walk away with the impression that every patient with that particular identity embodies those characteristics. ${ }^{10}$ The inclusion of any social identity should include a voice from that specific identity, such as the example "nothing about us without us". ${ }^{17}$ Pedagogies that utilize teams should also anticipate that any individual student may be looked at to speak for an entire population and may feel threatened via hostile actions or words. ${ }^{5}$ Therefore, the instructor should navigate difficult conversations or challenging moments in the classroom, and these strategies should focus on psychological safety of every member of the classroom community. By bringing together the content expert (instructor) and lived experiences, a stronger and more inclusive case can be built to better the learning opportunity for students. ${ }^{3}$

Potential biases and inaccurate portrayals of individuals must also be considered when identities are embedded in case discussions. These biases may be disguised as race corrections, such as calculators for estimated Glomerular Filtration Rate, Vaginal Birth after Cesarean, or in disease states such as heart failure, cardiothoracic surgery, organ transplant, urology, and oncology. The result of racial corrections may lead to poor health outcomes for patients who are non-White, most commonly Black. ${ }^{8}$ Interestingly, the guidelines and organizations that support these calculators are silent on their use in individuals who are biracial or have multiple racial identities,${ }^{7,8}$ leading to questions about the calculators' racial correction validity. Vyas et $\mathrm{al}^{8}$ are clear in advocating for inclusion of race in clinical care while remaining vigilant to its impact and inclusion in the care delivered. Importantly, visual presentation of a patient, such as race, could lead to further bias. An OSCE-based assessment demonstrated a significant reduction in the time to requesting an interpreter in standardized patients (SP) with limited English proficiency ${ }^{13}$; however, the authors do not comment on if the visualization of the SPs' race, not their abilities, contributed to the reduced time.

Finally, instructors may feel inadequately prepared to lead discussions regarding social identities. ${ }^{3}$ Instructors may hesitate to include aspects of patient social identities to avoid further stereotyping of identities. ${ }^{5}$ Instructors often anticipate facing roadblocks or tension within their program, particularly prior to best practices being established or communicated amongst instructors. ${ }^{3}$ These barriers could include lack of time within curricula, lack of instructor knowledge or comfort, or where to best integrate content. ${ }^{6}$ Students who embody a particular social identity may bring a higher level of knowledge into the discussions, ${ }^{4}$ which offers additional learning opportunities for students without that identity. Learners can also be an asset to identifying new resources for instructors to use, such as websites that host resources such as transgender care, cultural competence, and a provider listing for individuals who are gender nonbinary. ${ }^{18}$ Hesitating to include identities could construct a barrier for students, by limiting the practice of using the identities to care for patients with intersecting backgrounds. When gender minority SP were polled, they identified three themes of opportunities: personal connection, gaps in care received, and opportunities for improvement of medical education. ${ }^{5}$ Instructors and learners have responsibilities to education, through questioning content, accepting feedback and continuous improvement in a world with fluctuating definitions for identities. ${ }^{15}$ Ingraining social identities into health professional education can progress over time, as demonstrated by transgender care in physician assistant curricula, where $59-67 \%$ of programs documented its inclusion in 2014 to nearly $86 \%$ in $2020 .{ }^{6}$ Another approach may be to integrate inclusivity into admissions processes, as done within medical education. ${ }^{9}$

The AMA recently introduced a paper regarding reporting of race and ethnicity, with the intent of reducing unintentional bias in published literature. ${ }^{9}$ The authors position that excluding race and ethnicity in reporting disregards important social inequities and constructs that should be considered to remove barriers to care. They also acknowledge 
that including them in medical research remains important at this time; however, this statement is specific to published literature and not necessarily didactic cases. Reporting of race and ethnicity should be done as separate identities with acknowledgement that individuals may not adherence to just one. This is an important concept to consider in writing cases: human beings are much more complex than the one-or-two-word description of a patient, regarding race and ethnicity.

There are important limitations to note with this study. First, investigator interpretation was needed to determine the 'identity needed to answer case questions'. This interpretation could have led to investigator bias; to control for this, two investigators reviewed each case and discrepancies were resolved by a third, impartial investigators. Second, identities were only counted if explicitly named within the case, which could have led to underrepresented implicit identities. Third, patient cases were specific to this course series at one institution and are not representative of other curricula. This limits the generalizability of the findings. An important fourth consideration is that some identities were being examined at a high level within society at the time of the writing of this article, whereas the depths of these discussions were not outwardly occurring when this project was initiated. Another limitation is that the identities examined here are not an all-inclusive list to consider. Others could include language spoken and use of an interpreter, or immigration or citizen status. A final limitation of this study, as well as of other published data, ${ }^{16}$ is that the intersection of these social identities is difficult to measure. For example, the perceived value of learning by including a patient who is Black versus a female patient who also identifies as Black and LGBTQIA with a lower socioeconomic status may not always be accurately measured. Though not unique to this type of scholarship, these intersections were not addressed.

\section{CONCLUSION}

This study found eight social identities were rarely explicitly included in pharmacotherapeutics cases presented to one cohort of students at a private, PWI in the Midwest. Thoughtful and purposeful inclusion of social identities is critical to inclusive and individualized care practices with student pharmacists. It is important to consider that cultural competence is not a checklist, and it should evolve as practitioners navigate their fields. ${ }^{19,20}$ Doing this work in the classroom allows for inclusivity and individualization to be carried into professional practices, though it is not the only opportunity for exposure. Collaboration amongst pharmacy programs would allow comparison of techniques used, the effect of these interventions on the inclusion of social identities, and student perceptions or knowledge-gained by students. In determining the next steps in including social identities within didactic teaching, involving cases, institutions first need to perform a self-assessment of the current level of inclusion.

\section{ACKNOWLEDGEMENTS}

The authors would like to thank our colleagues involved in the course series, and Erica Ottis and Eliza DyBoarman for their thoughtful contributions and inspiration. Additionally, thanks to Anna Marceau for their contributions to resources for educators.

\section{REFERENCES}

1. Braveman P, Gottlieb L. The social determinants of health: it's time to consider the causes of the causes. Public Health Rep. 2014;129 Suppl 2(Suppl 2):19-31.

2. Sample cultural identifiers. National Association of Independent Schools. https://www.nais.org/articles/pages/sample-cultural-identifiers/ Accessed June 29, 2021

3. Bohnert CA, Combs RM, Noonan EJ, Weathers AE, Weingartner LA. Gender minorities in simulation: a mixed methods study of medical school standardized patient programs in the United States and Canada. Simul Healthc. 2002 Dec 2; online ahead of print. DOI: 10.1097/SIH.0000000000000532.

4. Jamieson A, Cross H, Arthur S, Nambiar K, Llewellyn CD. Patient sexual orientation and gender identity disclosure. Clin Teach 2020;17:669-73. DOI: 10.1111/tct/13182

5. Noonan EJ, Weingartner LA, Combs RM, Bohnert C, Shaw MA, Sawning S. Perspectives of transgender and genderqueer standardized patients. Teach Learn Med 2021;33(2):116-28. DOI: 10.1080/10401334.2020.1811096

6. Rolls J, Davis J, Backman R, Wood T, Honda T. Curricular approaches to transgender health in physician assistant education. Academic Med 2020;95(10):1563-9.

7. Amutah C, Greenidge K, Mante A, Munyikwa M, Surya SL, Higginbotham E, Jones DS, Lavizzo-Mourey R, Roberts D, Tsai J, Aysola J. Misrepresenting race - the role of medical schools in propagating physician bias. $\mathrm{N}$ Engl J Med 2021;384(9):872-8. DOI: 10.1056/NEJMms2025768

8. Vyas DA, Eisenstein LG, Jones DS. Hidden in plain sight - reconsidering the use of race correction in clinical algorithms. N Engl J Med 2020;383(9):874-82. DOI: 10.1056/NEJMms2004740 
9. Flanagin A, Frey T, Christiansen SL, et al. Updated guidance on the reporting of race and ethnicity in medical and science journals. JAMA. 2021;326(7):621-7.

10. Miller E, Green AR. Student reflections on learning cross-cultural skills through a 'cultrual competence' OSCE. Medical Teacher 2007;29:e76-84.

11. QuickFacts Des Moines. United States Census Bureau. https://www.census.gov/quickfacts/fact/table/desmoinescityiowa,IA/PST045219 Published 2019. Accessed March 22, 2021.

12. QuickFacts Iowa. United States Census Bureau. https://www.census.gov/quickfacts/IA Published 2019. Accessed March 22, 2021.

13. Fune J, Chinchilia JP, Hoppe A, Bganugo C, et al. Lost in translation: an OSCE-based workshop for helping learners navigate a limited English proficiency patient encounter. MedEdPORTAL. 2021;17:1118. DOI: 10.15766/mep_2374-8265.11118

14. Ripp K, Braun L. Race/Ethnicity in medical education: an analysis of a question bank for Step 1 of the United States Medical Licensing Examination. Teach Learn Med 2017;29(2):115-22. DOI: 10.1080/10401334.2016.1268056

15. Finucane TE. Mention of a patient's "race" in clinical presentations. Virtual Mentor; Am Med Assoc J Ethics 2014;16(6):423-7.

16. Bochatay N, Bajwa NM, Ju M, Applebaum NP, van Schaik SM. Towards equitable learning environments for medical education: bias and the intersection of social identities. Med Educ 2021 Jul 26. DOI: 10.1111/medu. 14602

17. Iezzoni LI, Long-Bellil LM. Training physicians about caring for persons with disabilities: "Nothing about us without us!". Disabil Health J. 2012;5(3):136-9. DOI: 10.1016/j.dhjo.2012.03.003

18. GLMA Health Professional Advancing LGBTQ Equality. Available at: http://www.glma.org . Accessed on November 3, 2021.

19. Balzora S, Abiri B, Wang XJ, McKeever J, Poles M, Zabar S, et al. Assessing cultural competency skills in gastroenterology fellowship training. World J Gastroenterol. 2015;21(6):1887-92. DOI: 10.3748/wjg.v21.i6.1887

20. Lee YH, Lin SC, Wang PY, Lin MH. Objective structural clinical examination for evaluating learning efficacy of Cultural Competence Cultivation Programme for nurses. BMC Nursing. 2020;19(114):1-8. DOI: 10.1186/s12912020-00500-3.

Table 1. Descriptions of the eight social identities reviewed ${ }^{2}$

\section{Social Identity}

Ability

Ethnicity

Gender Identity

Nationality

Race

Religion/Spirituality

Sexual Orientation

Socioeconomic Status

\author{
Description \\ The physical or mental capacity to do or perform a task \\ A person of group of people who share a national, \\ cultural, and/or linguistic heritage \\ Who a person sees themselves as (eg, a woman, man, \\ transgender, non-binary, or as none of these) \\ The individual's country of origin \\ A group that is socially defined based on physical \\ criteria (eg, skin color and facial features) \\ An institutionalized or personal system of beliefs and \\ practices relating to the divine \\ An emotional, romantic, sexual, spiritual, affectional, \\ and/or relational attraction to another person(s) \\ The social standing based on income or positions in \\ society (eg, working class, middle class, upper class)
}


Table 2. Social Identity Inclusion in Pharmacotherapy Cases

\begin{tabular}{|c|c|c|}
\hline & Overall Cases $(n=144)$ & \\
\hline Social Identity & Present in case $(n, \%)^{a}$ & Needed to answer questions $(n, \%)^{a}$ \\
\hline Race & $21(14.6)$ & $11(7.6)$ \\
\hline Socioeconomic status & $9(6.3)$ & $17(11.8)$ \\
\hline Ability & $7(4.9)$ & $13(9.0)$ \\
\hline Gender Identity & $2(1.4)$ & $27(18.8)$ \\
\hline Sexual orientation & $2(1.4)$ & $1(0.7)$ \\
\hline Nationality & $2(1.4)$ & $1(0.7)$ \\
\hline Ethnicity & $0(0)$ & $\mathrm{n} / \mathrm{a}^{\mathrm{b}}$ \\
\hline Religion & $0(0)$ & $\mathrm{n} / \mathrm{a}^{\mathrm{b}}$ \\
\hline
\end{tabular}

